\title{
Coordinated Microanalyses of Seven Particles of Probable Interstellar Origin from the Stardust Mission.
}

Andrew J. Westphal, ${ }^{1 *}$ Rhonda M. Stroud, ${ }^{2}$ Hans A. Bechtel, ${ }^{3}$ Frank E. Brenker, ${ }^{4}$ Anna L. Butterworth, ${ }^{1}$ George J. Flynn, ${ }^{5}$ David R. Frank, ${ }^{6}$ Zack Gainsforth, ${ }^{1}$ Jon K. Hillier, ${ }^{7}$ Frank Postberg, ${ }^{7}$ Alexandre S. Simionovici, ${ }^{8}$ Veerle J. Sterken, ${ }^{9}$ Carlton Allen, ${ }^{10}$ David Anderson, ${ }^{1}$ Asna Ansari, ${ }^{11}$ Sa sa Bajt, ${ }^{12}$ Ron K. Bastien, ${ }^{6}$ Nabil Bassim, ${ }^{2}$ John Bridges, ${ }^{13}$ Donald E. Brownlee, ${ }^{14}$ Mark Burchell, ${ }^{15}$ Manfred Burghammer, ${ }^{16,18}$ Hitesh Changela, ${ }^{17}$ Peter Cloetens, ${ }^{18}$ Andrew M. Davis, ${ }^{19}$ Ryan Doll, ${ }^{20}$ Christine Floss, ${ }^{20}$ Eberhard Gru“n, ${ }^{21}$ Philipp R. Heck, ${ }^{11}$ Peter Hoppe, ${ }^{22}$ Bruce Hudson, ${ }^{23}$ Joachim Huth, ${ }^{22}$ Anton Kearsley, ${ }^{24}$ Ashley J. King, ${ }^{11}$ Barry Lai, ${ }^{25}$ Jan Leitner, ${ }^{22}$ Laurence Lemelle, ${ }^{26}$ Ariel Leonard, ${ }^{20}$ Hugues Leroux, ${ }^{27}$ Robert Lettieri, ${ }^{1}$ William Marchant, ${ }^{1}$ Larry R. Nittler, ${ }^{28}$ Ryan Ogliore, ${ }^{29}$ Wei Jia Ong, ${ }^{20}$ Mark C. Price, ${ }^{15}$ Scott A. Sandford, ${ }^{30}$ Juan-Angel Sans Tresseras, ${ }^{18}$ Sylvia Schmitz, ${ }^{4}$ Tom Schoonjans, ${ }^{16}$ Kate Schreiber, ${ }^{20}$ Geert Silversmit, ${ }^{16}$ Vicente A. Solé, ${ }^{18}$ Ralf Srama, ${ }^{31}$ Frank Stadermann, ${ }^{20 * *}$ Thomas Stephan, ${ }^{19}$ Julien Stodolna, ${ }_{1}^{1}$ Stephen Sutton, ${ }^{25}$ Mario Trieloff, ${ }^{7}$ Peter Tsou, ${ }^{32}$ Tolek Tyliszczak, ${ }^{3}$ Bart Vekemans, ${ }_{16}$ Laszlo Vincze, ${ }^{16}$ Joshua Von Korff, ${ }^{1}$ Naomi Wordsworth, ${ }^{33}$ Daniel Zevin, ${ }^{1}$ Michael E. Zolensky, ${ }^{10} 30714$ Stardust@home dusters ${ }^{34}$

${ }^{1}$ Space Sciences Laboratory, U. C. Berkeley, Berkeley, CA USA, ${ }^{2}$ Materials Science and Technology Division, Naval Research Laboratory, Washington, DC USA, ${ }^{3}$ Advanced Light Source, Lawrence Berkeley Laboratory, Berkeley, CA USA, ${ }^{4}$ Geoscience Institute, Goethe University Frankfurt, Frankfurt, Germany, ${ }^{5}$ SUNY Plattsburgh, Plattsburgh, NY USA, ESCG, NASA JSC, Houston, TX USA, ${ }^{7}$ Institut für Geowis- senschaften, University of Heidelberg, Germany, ${ }^{8}$ Institut des Sciences de la Terre, Observatoire des Sciences de 1'Univers de Grenoble, Grenoble, France, ${ }^{9}$ IRS, University Stuttgart, Stuttgart, IGEP, TU Braunschweig, Braunschweig, Germany and MPIK, Heidelberg, Germany, ${ }^{10}$ ARES, NASA JSC, Houston, TX USA, ${ }^{11}$ Field Museum of Natural History, Chicago, IL USA, ${ }^{12}$ DESY, Hamburg, Germany, ${ }^{13}$ Space Research Centre, University of Leicester, Leicester, UK, ${ }^{14}$ Department of Astronomy, University of Washington, Seattle, WA USA, ${ }^{15}$ University of Kent, Canterbury, Kent, UK, ${ }^{16}$ University of Ghent, Ghent, Belgium, ${ }^{17}$ University of New Mexico, ${ }^{18}$ European Synchrotron Radiation Facility, Grenoble, France, ${ }^{19}$ University of Chicago, Chicago, IL USA, ${ }^{20}$ Washington University, St. Louis, MO USA, ${ }^{21}$ Max-Planck-Institut für Kernphysik, Heidelberg, Germany, ${ }^{22}$ Max-Planck-Institut für Chemie, Mainz, Germany, ${ }^{23} 615$ William St., Apt 405, Midland, Ontario, Canada, ${ }^{24}$ Natural History Museum, London, UK, ${ }^{25}$ Advanced Photon Source, Argonne National Laboratory, Lemont, IL USA, ${ }^{26}$ Ecole Normale Superieure de Lyon, Lyon, France, ${ }^{27}$ University Lille 1, France, ${ }^{28}$ Carnegie Institution of Washington, Washington, DC USA, ${ }^{29}$ University of Hawai'i at Manoa, Honolulu, HI USA, ${ }^{30}$ NASA Ames Research Center, Moffett Field, CA USA, ${ }^{31}$ IRS, University Stuttgart, Stuttgart, Germany, ${ }^{32}$ Jet Propulsion Laboratory, Pasadena, CA USA, ${ }^{33}$ Wexbury, Farthing Green Lane, Stoke Poges, South Buckinghamshire, UK, ${ }^{34}$ Worldwide

Stardust, a NASA Discovery-class mission, was the first sample-return mission to return solid samples from beyond the Moon. Stardust was effectively two missions in one spacecraft: it returned the first materials from a known primitive solar system body, the Jupiter-family comet Wild 2; Stardust also returned a collector that was exposed to the contemporary interstellar dust stream for 200 days during the interplanetary cruise. Both collections present severe technical challenges in sample preparation and in analysis. By far the largest collection is the cometary one: approximately $300 \mu \mathrm{g}$ of material was returned from Wild 2, mostly consisting of $\sim 1 \mathrm{ng}$ particles embedded in aerogel or captured as residues in craters on aluminum foils. Because of their relatively large size, identification of the impacts of 
cometary particles in the collection media is straightforward. Reliable techniques have been developed for the extraction of these particles from aerogel. Coordinated analyses are also relatively straightforward, often beginning with synchrotron-based x-ray fluorescence (S-XRF), X-ray Absorption Near-Edge Spectroscopy (XANES) and x-ray diffraction (S-XRD) analyses of particles while still embedded in small extracted wedges of aerogel called "keystones", followed by ultramicrotomy and TEM, Scanning Transmission X-ray Microscopy (STXM) and ion microprobe analyses (e.g., [1]). Impacts in foils can be readily analyzed by SEM-EDX, and TEM analysis after FIB liftout sample preparation.

In contrast, the interstellar dust collection is vastly more challenging. The sample size is approximately six orders of magnitude smaller in total mass. The largest particles are only a few pg in mass, of which there may be only $\sim 10$ in the entire collection. The technical challenges, however, are matched by the scientific importance of the collection. We formed a consortium - the Stardust Interstellar Preliminary Examination (ISPE) - to carry out an assessment of this collection, partly in order to characterize the collection in sufficient detail so that future investigators could make well-informed sample requests. The ISPE is the sixth PE on extraterrestrial collections carried out with NASA support. Some of the basic questions that we asked were: how many impacts are there in the collector, and what fraction of them have characteristics consistent with extraterrestrial materials? What is the elemental composition of the rock-forming elements? Is there crystalline material? Are there organics?

Here we present coordinated microanalyses of particles captured in aerogel, using S-FTIR [5], S-XRF [7-9], STXM [6], S-XRD [10]; and coordinated microanalyses of residues in aluminum foil [13], using SEM-EDX, Auger spectroscopy, STEM, and ion probe. We discuss a novel approach that we employed for identification of tracks in aerogel [3], and new sample preparation techniques developed during the ISPE [4]. We have identified seven particles - three in aerogel and four in foils - that are most consistent with an interstellar origin. The seven particles exhibit a large diversity in elemental composition [2]. Dynamical evidence, supported by laboratory simulations of interstellar dust impacts in aerogel and foils [11], and numerical modeling of interstellar dust propagation in the heliosphere [12], suggests that at least some of the particles have high optical cross-section, perhaps due to an aggregate structure. However, the observations are most consistent with a variety of morphologies.

\section{References:}

[1] R Ogliore et al, ApJ 745 (2012), p. 19

[2] A. J. Westphal et al., Meteorit. Planet. Sci. (2014a) in press.

[3] A. J. Westphal et al., Meteorit. Planet. Sci. (2014b) in press.

[4] D. R. Frank et al., Meteorit. Planet. Sci. (2014) in press.

[5] H. A. Bechtel et al., Meteorit. Planet. Sci. (2014) in press.

[6] A. L. Butterworth et al., Meteorit. Planet. Sci. (2014) in press.

[7] F. E. Brenker et al., Meteorit. Planet. Sci. (2014) in press.

[8] A. S. Simionovici et al., Meteorit. Planet. Sci. (2014) in press.

[9] G. J. Flynn et al., Meteorit. Planet. Sci. (2014) in press.

[10] Z. Gainsforth et al., Meteorit. Planet. Sci. (2014) in press.

[11] F. Postberg et al., Meteorit. Planet. Sci. (2014) in press.

[12] V. J. Sterken et al., Meteorit. Planet. Sci. (2014) in press.

[13] R. M. Stroud et al., Meteorit. Planet. Sci. (2014) in press. 